Article

\title{
A Distributed Energy Resources Aggregation Model Based on Multi-Scenario and Multi-Objective Methodology
}

\author{
Hong Li ${ }^{1}$, Jie Duan ${ }^{1, *}$, Dengyue Zhang ${ }^{1}$ and Jinghui Yang ${ }^{1,2}$ \\ 1 Department of Electrical Engineering, North China Electric Power University, Baoding 071003, China \\ 2 Department of Electrical and Electronic Engineering, University of Bristol, Bristol BS15QD, UK \\ * Correspondence: duanjie@ncepu.edu.cn
}

Received: 25 July 2019; Accepted: 22 August 2019; Published: 2 September 2019

check for updates

\begin{abstract}
Aggregation technology can integrate distributed energy resources (DERs) into resource aggregation (RA) to achieve efficient utilization of resources. This paper studies a DERs aggregation model to construct a RA. Firstly, considering the uncertainty of the output of distributed generation (DG), the characteristics of DG are analyzed and the daily eigenvalues are extracted. The contour coefficient is introduced and the improved K-means algorithm is used to cluster the daily eigenvectors to get the multiple probability scenarios in a single season. Then, in order to obtain a RA with lower daily average cost, better power generation characteristics and higher regional aggregation degree, the DERs aggregation model based on multi-scenario and multi-objective is established considering multiple constraints. To obtain a compromise optimal solution, the cellular bat algorithm based on fuzzy membership degree (FMD-CBA) is used to solve the model. Finally, the validity of the multi-scenario and multi-objective model in a single season is verified by an example.
\end{abstract}

Keywords: distributed energy resources; cluster analysis; multi-objective optimization; scenario division; cellular bat algorithm

\section{Introduction}

With the increasing demand of electricity in the world and the serious problems of the energy shortage, environmental pollution and climate change, the shortcomings of traditional power generation are increasingly obvious [1,2]. In this situation, distributed energy resources (DERs) have developed rapidly [3-5]. DERs are composed of distributed generation (DG), demand resource (DR), energy storage (ES), etc. DERs can improve the flexibility of the power system [6]. However, DERs are scattered, diverse and different in characteristics. It is difficult for a single DER to participate in the operation of the power market, so the resource aggregation technology has emerged [7-9]. In order to realize optimal allocation and efficient utilization of resources, aggregation can integrate DERs into the RA with simple and flexible regulation by certain technologies. Furthermore, RA can participate in the power market and grid operation as a whole. This paper aims to construct a RA by studying the resource aggregation model and improve resource utilization.

The uncertainty of DG output should be considered when establishing the aggregation model. In [10], in order to deal with the uncertainty of renewable energy, a two-stage stochastic programming model is established. The K-means clustering algorithm is used to reduce the number of scenarios while maintaining the correlation between uncertain data. In [11], a scenario generation method based on Wasserstein distance measure and K-means clustering scenario reduction technology is proposed, which transforms the stochastic problem into the deterministic problem. In [12], the whole scenario sets are generated by discretizing the output of each time, and then the probability distance is used to reduce the scenario sets. 
RA is the aggregation of DERs, and there are different objective functions of the aggregation model. In [13], considering that ES has a great impact on the economy of RA, a two-level fuzzy stochastic expectation model is proposed to optimize the cost of ES, and the model is verified on a modified IEEE-33 test feeder. In [14], considering the comfort requirement and cost of DR, a mixed-integer linear optimization model is established, and the model can compute the optimal scheduling of a prosumer's assets. In [15], a strategy of load aggregation for central air conditioning is proposed to minimize the operating cost of load aggregator, and the particle swarm optimization algorithm is adopted to solve the optimal problem. In [16], considering the charging characteristics of EV, the aggregation of EV is realized with the objective of maximizing the profit of load aggregator. In [17], a mixed-integer linear programming model is established to maximize the profits of the virtual power plant, and the advantage of using the model to devise offering strategies becomes more apparent as the stochastic parameters become increasingly accurate.

When using the clustering algorithm and multi-scenario to establish the model of DG output, the cluster object is 365 output curves in the whole year. The dimension of clustering vectors is generally 24, that is, the number of hours in a day [10-12]. For DG in multiple regions, the following issues are considered in this paper: (1) The dimension of clustering vectors will multiply with the increase of the number of regions, which can increase the complexity of the calculation. (2) The single aggregation scheme obtained in a longer time scale cannot achieve good resource aggregation effect. (3) It is necessary to objectively determine the cluster number. When establishing the aggregation models, most references focused on aggregating DERs of the same kind $[13,14,16]$. Although many kinds of DERs have been studied in some references, the objective function of the aggregation model is also simple $[15,17]$. In the process of establishing an aggregation model, the following issues are considered in this paper: (1) Several realistic objective functions and constraints need to be proposed. (2) It is necessary to find an appropriate optimization algorithm to solve the model.

According to the existing research results and shortcomings of the above references, this paper has made some improvements. When clustering the output curves of DG, the daily eigenvectors composed of the daily eigenvalues are used to replace the daily average output curves, which can reduce the complexity of the calculation, and the daily eigenvectors can represent the output characteristics of all regions. The time scale is set as the season, which can improve the effect of resource aggregation. This paper takes spring as an example to verify the effectiveness of the aggregation model based on single-season and multi-scenario methodology. Considering the geographical location and resource characteristics of DERs, this paper proposes the regional aggregation degree and establishes a multi-objective aggregation model.

Therefore, considering the seasonal difference of DG output, this paper establishes a DERs aggregation model based on multi-scenario and multi-objective in a single-season time scale. Firstly, the daily eigenvectors of DG are extracted, the contour coefficient is introduced, and multiple probability scenarios are obtained according to the improved K-means algorithm. Then a multi-objective DERs aggregation model is established based on multi-scenario, and the model is solved by the cellular bat algorithm based on fuzzy membership degree. Finally, an example is given to verify the validity of the model.

\section{DERs Multi-Scenario Construction}

\subsection{Daily Eigenvectors of DG}

DERs are composed of DG, DR and ES. Wind power (WP) and photovoltaic (PV) are influenced by wind speed and solar irradiance all year round. The output has the characteristics of randomness, fluctuation and intermittence, but it also has certain regularity. The output of WP and PV has obvious seasonality. If each season is regarded as a scenario directly, the accuracy of scenario division is insufficient. Therefore, this paper uses the clustering algorithm to generate multi-scenario for each season to improve the accuracy of the model. Firstly, the daily eigenvalues representing the 
characteristics of WP and PV output are extracted. Then, the daily eigenvectors composed of the daily eigenvalues of all regions are used as the clustering objects, and the initial scenarios are reduced according to the improved K-means algorithm. The daily characteristic values of WP and PV output curves include daily average, daily maximum, daily peak time and daily peak-valley difference. If $n$ is the number of aggregated regions and $d$ is the date, the day eigenvector is as follows:

$$
A(d)=\left(A_{1}^{W P}, A_{2}^{W P}, \ldots, A_{n_{W P}}^{W P}, A_{1}^{P V}, A_{2}^{P V}, \ldots, A_{n_{P V}}^{P V}\right)
$$

where $A_{i}^{\mathrm{WP}}=\left(a_{i, 1}^{W P}, a_{i, 2}^{\mathrm{WP}}, a_{i, 3}^{\mathrm{WP}}, a_{i, 4}^{\mathrm{WP}}\right), A_{i}^{P V}=\left(a_{i, 1}^{P V}, a_{i, 2}^{P V}, a_{i, 3}^{P V}, a_{i, 4}^{P V}\right), A_{i}^{W P}$ is the daily eigenvector of WP in the $i$-th region, $A_{i}^{P V}$ is the daily eigenvector of $P V$ in the $i$-th region, $a_{i, 1}, a_{i, 2}, a_{i, 3}$ and $a_{i, 4}$ represent the daily average, daily maximum, daily peak time and daily peak-valley difference in the $i$-th region, respectively.

\subsection{Generation of Multi-Scenario}

If there are $D$ days in a season, the number of day eigenvectors in each season is $D$, and the day eigenvector is a row vector. In this paper, the improved K-means algorithm is used to cluster the daily eigenvectors to achieve the purpose of scenario reduction.

$\mathrm{K}$-means is an iterative clustering algorithm which is implemented by continuously updating the cluster center and assigning objects to each class [18]. In order to determine the optimal clustering number $\mathrm{K}$, the contour coefficient is introduced to evaluate the clustering results [19]. The value range of contour coefficient is $[-1,1]$. The closer to 1 , the better the cohesion and separation are. The contour coefficient of the $i$-th cluster vector is as follows:

$$
s(i)=\frac{b_{t e r}(i)-b_{\text {tra }}(i)}{\max \left\{b_{\text {tra }}(i), b_{\text {ter }}(i)\right\}}
$$

where $b_{\text {tra }}(i)$ is the intra-cluster dissimilarity of the $i$-th cluster vector, $b_{\text {ter }}(i)$ is the inter-cluster dissimilarity of the $i$-th cluster vector. The steps of generating multi-scenario by the improved K-means algorithm are as follows:

(1) Initialize the day eigenvectors and the number of clusters. The number of day eigenvectors is $D$, and the number of clusters $K$ is initialized to 2 .

(2) Select $K$ clustering centers from $D$ clustering objects randomly, and record them as $c_{1,0}, c_{2,0}, \ldots c_{k, 0}$.

(3) For any cluster object, calculate the Euclidean distance to each cluster center, and classify the object into the cluster center with the shortest distance.

(4) Use the average method to update the clustering centers of each class of clustering objects. The new clustering centers are recorded as $c_{1}, c_{2}, \ldots c_{k}$. If $\max \left\|c_{i, 0}-c_{i}\right\| \leq \delta$, take to the next step, otherwise, update the clustering centers and return to (3). Where $\delta$ is the threshold of the iterative termination.

(5) Calculate the contour coefficients corresponding to the $D$ clustering objects, and take the average of $D$ contour coefficients as the contour coefficient of clustering results. The contour coefficient is recorded as $S_{k}$.

(6) $K=K+1$, if $K>9$, take to the next step, otherwise return to (2).

(7) Find the minimum value $S_{k . m i n}$ of the contour coefficients of the clustering results. Then the $K$ corresponds to the final clustering result, and the probability of scenario is determined according to the number of clustering objects in each class, which is recorded as $P_{k}$.

This paper reduces the computational complexity by reducing the dimension of clustering vectors. The dimension of clustering vectors consisting of daily output curve is $n \times 24 \times 2$, and the dimension of clustering vectors consisting of daily eigenvalues is $n \times 4 \times 2$. In the above, $n$ is the number of aggregated regions, 24 is the number of hours in a day, 4 is the number of daily eigenvalues, and 2 
is the number of resources. The dimension of the clustering vectors of the latter is $1 / 6$ of that of the former, so using daily eigenvectors for clustering analysis can reduce the computational complexity.

\section{Aggregation Model Based on Multi-Scenario and Multi-Objective}

\subsection{Objective Functions}

In order to deal with the uncertainty when establishing the aggregation model, this paper describes the output of WP and PV through multiple probability scenarios.

(1) The lowest daily average cost of RA

The daily average cost is mainly composed of generation cost, energy storage cost and response cost, which correspond to DG, ES and DR in DERs respectively. The objective function is as follows:

$$
\min f_{1}=\sum_{k=1}^{K} p_{k} \sum_{n=1}^{N} C_{n, k}^{D G} X_{n}^{D G}+\sum_{n=1}^{N} C_{n}^{E S} X_{n}^{E S}+\sum_{n=1}^{N} C_{n}^{D R} X_{n}^{D R}
$$

where $K$ is the number of multiple scenarios, $N$ is the number of aggregated regions, $P_{k}$ is the probability of the $k$-th scenario, $C_{n, k}^{D G}$ is the average daily generation cost of DG in the $n$-th region under the $K$-th scenario, $X_{n}^{D G}$ represents whether there is DG in the $n$-th region, which is 1 and vice versa, $C_{n}^{E S}$ is the charge-discharge cost of energy storage in the $n$-th region, $X_{n}^{E S}$ represents whether there is ES in the $n$-th region, $C_{n}^{D R}$ is the response cost of DR in the $n$-th region, and $X_{n}^{D R}$ represents whether there is DR in the $n$-th region.

DG includes WP and PV, and its cost mainly includes investment cost and operation- maintenance costs [20]. The generation cost is as follows:

$$
C_{n}^{D G}(k)=\sum_{t=1}^{T}\left(1+M_{n}^{D G}\right) \frac{\beta_{n}^{D G} I_{n}^{D G}}{P_{t, n, k}^{D G}}
$$

where $t$ is the time series with an interval of $1 \mathrm{~h}, M_{n}^{D G}$ is the maintenance rate of DG in the $n$-th region, $\beta_{n}^{D G}$ is the annual investment rate of DG in the $n$-th region, $I_{n}^{D G}$ is the initial investment of DG in $n$-th region, $P_{t, n, k}^{D G}$ is the DG output at the $t$-time in the $n$-th region under the $K$-th scenario.

The energy storage cost is expressed as the product of the battery loss rate $\gamma_{d}$ of the unit power and the actual charge-discharge power:

$$
C_{n}^{E S}=\sum_{t=1}^{T} \gamma_{d} P_{t, n}^{E S}
$$

where $\gamma_{d}$ is the battery loss rate of the unit power, $P_{t, n}^{E S}$ is the charge-discharge power at the $t$-time in the $n$-th region.

When users actively participate in demand response and reduce the load at a specific time according to the agreement requirements, the company will provide compensation to users [21]. The response cost is as follows;

$$
C_{n}^{D R}=\sum_{t=1}^{T} c_{n} P_{t, n}^{D R}
$$

where $c_{n}$ is the compensation cost of DR in the $n$-th region, $P_{t, n}^{D R}$ is the response capacity at the $t$-time in the $n$-th region. 
(2) The optimal generation characteristics of RA

Daily average output and curve volatility are used to measure the generation characteristics of RA. DG belongs to generation resources; although DR cannot generate electricity directly, it can reduce load and provide the virtual output at a specific time; ES is the energy-consuming resources for RA. The objective function of the maximum daily average output is as follows:

$$
\max f_{2}=\sum_{k=1}^{K} p_{k} \sum_{t=1}^{T} \sum_{n=1}^{N} P_{t, n, k}^{D G} X_{n}^{D G}-\sum_{t=1}^{T} \sum_{n=1}^{N} P_{t, n}^{E S} X_{n}^{E S}+\sum_{t=1}^{T} \sum_{n=1}^{N} P_{t, n}^{D R} X_{n}^{D R}
$$

The curve $L$ is used to represent the difference between the virtual output curve of RA and the electric load curve. The volatility of curve $L$ is defined as the ratio of the standard deviation of the output force to the geometric mean at each time [22]. The smaller the fluctuation rate is, the smoother the curve $L$ is, the better the fitting degree between the virtual output curve and the electric load curve is. The objective function is as follows:

$$
\left\{\begin{array}{l}
\min f_{3}=V_{L}=\frac{\sigma}{\mu}=\frac{\sqrt{\frac{1}{T} \sum_{t=1}^{T}\left(L_{t}-\bar{L}\right)^{2}}}{\sqrt[T]{\prod_{t=1}^{T} L_{t}}} \\
L=\sum_{k=1}^{K} p_{k} \sum_{n=1}^{N} P_{n, k}^{D G} X_{n}^{D G}-\sum_{n=1}^{N} P_{n}^{E S} X_{n}^{E S}+\sum_{n=1}^{N} P_{n}^{D R} X_{n}^{D R}-P_{0}
\end{array}\right.
$$

where $L$ is the curve to measure volatility, $V_{L}$ is the volatility of curve $L, \sigma$ is the standard deviation of the curve $L, \mu$ is the geometric mean of the curve $L$.

(3) The highest aggregation degree of RA

When choosing DERs in each region, the geographical location of the region and the resource characteristics need to be considered. The distance between the $n$-th region and the center is defined as follows:

$$
d(n)=\sqrt{\left(x_{n}-x_{0}\right)^{2}+\left(y_{n}-y_{0}\right)^{2}}
$$

where $\left(x_{0}, y_{0}\right)$ is the location of the geometric center of the whole region, $\left(x_{n}, y_{n}\right)$ is the location of the $n$-th region.

The average resource density in the $n$-th region is defined as follows;

$$
\rho(n)=\frac{\sum_{k=1}^{K} p_{k} \sum_{t=1}^{T} P_{t, n, k}^{D G} X_{n}^{D G}+\sum_{t=1}^{T} P_{t, n}^{E S} X_{n}^{E S}+\sum_{t=1}^{T} P_{t, n}^{D R} X_{n}^{D R}}{S_{n}}
$$

where $S_{n}$ is the area of the $n$-th region.

When constructing a RA, the more centralized the location of DERs is, the easier it is to regulate and control. In this paper, the aggregation degree of RA is calculated by combining the geographic location and average resource density of each region. The objective function of the highest aggregation degree is as follows;

$$
\min f_{4}=\sum_{n=1}^{N} d(n) \rho(n)
$$

In summary, the optimal objective function set of the DERs aggregation model is as follows;

$$
F=\left(\min f_{1}, \max f_{2}, \min f_{3}, \min f_{4}\right)
$$


$F$ represents the minimum daily average cost, maximum daily output, minimum curve volatility and the highest aggregation degree of the RA. The decision variable $X_{n}$ of the $n$-th region representing the presence or absence of various resources is as follows;

$$
X_{n}=\left[X_{n}^{D G}, X_{n}^{E S}, X_{n}^{D R}\right]^{T}
$$

The DERs aggregation model established in this paper is a multi-objective 0-1 planning model. The decision variable of all regions is a matrix composed of 0 and 1 elements;

$$
\left(X_{1}, X_{2}, \ldots, X_{n-1}, X_{n}\right)=\left[\begin{array}{ccccc}
X_{1}^{D G} & X_{2}^{D G} & \ldots & X_{n-1}^{D G} & X_{n}^{D G} \\
X_{1}^{E S} & X_{2}^{E S} & \ldots & X_{n-1}^{E S} & X_{n}^{E S} \\
X_{1}^{D R} & X_{2}^{D R} & \ldots & X_{n-1}^{D R} & X_{n}^{D R}
\end{array}\right]
$$

\subsection{Constraints}

(1) The scale constraints of RA

RA can aggregate DG, ES and DR into a whole, and the number of resources for constructing a RA should not be too small:

$$
\sum_{n=1}^{N}\left(X_{n}^{D G}+X_{n}^{E S}+X_{n}^{D R}\right) \geq X_{\min }
$$

where $X_{\min }$ is the minimum number of resources.

(2) Response capacity constraints

RA can participate in the ancillary service market and the electricity market, and its response capacity should not be too small;

$$
\sum_{k=1}^{K} p_{k} \sum_{t=1}^{T} \sum_{n=1}^{N} P_{t, n}^{D G}(k) X_{n}^{D G}+\sum_{t=1}^{T} \sum_{n=1}^{N} P_{t, n}^{D R} X_{n}^{D R} \geq Q_{\min }
$$

where $Q_{\min }$ is the minimum response capacity.

(3) WP and PV utilization constraints

In the process of aggregation, WP and PV should be utilized to the greatest extent to improve the utilization of resources:

$$
\frac{\sum_{k=1}^{K} p_{k} \sum_{t=1}^{T} \sum_{n=1}^{N} P_{t, n}^{D G}(k) X_{n}^{D G}+\sum_{t=1}^{T} \sum_{n=1}^{N} P_{t, n}^{E S} X_{n}^{E S}+\sum_{t=1}^{T} \sum_{n=1}^{N} P_{t, n}^{D R} X_{n}^{D R}}{\sum_{k=1}^{K} p_{k} \sum_{t=1}^{T} \sum_{n=1}^{N} P_{t, n}^{D G}(k)+\sum_{t=1}^{T} \sum_{n=1}^{N} P_{t, n}^{E S}+\sum_{t=1}^{T} \sum_{n=1}^{N} P_{t, n}^{D R}} \geq \eta_{\min }
$$

where $\eta_{\min }$ is the minimum resource utilization rate.

\section{Cellular Bat Algorithm Based on Fuzzy Membership Degree}

\subsection{Cellular Automata Principle}

Cellular automata have the characteristics of simple regularity of components, the locality between units, high parallelism of information processing and complex globality. It is an effective tool for exploring complex systems [23]. The cellular automata can be expressed as follows;

$$
E=\left(L_{D}, S, M, f\right)
$$


where $E$ is a cellular automaton system, $L$ is a cellular space, $D$ is the dimension of the cellular space, $S$ is the state set of cells, $M$ is a set of all cells in a neighborhood, $f$ is a local update rule.

The evolution process of cellular automata can be regarded as the change of state in time. If the cellular state set on integer set is recorded as $S^{Z}$, the dynamic evolution rule is expressed as follows:

$$
F: S_{t}^{Z} \rightarrow S_{t+1}^{Z}
$$

The dynamic evolution rule is determined by the local updating rule $f$ of each cell. In the $d$-dimensional space, the cellular neighbor is recorded as $S^{r}, r$ is the radius of the cellular neighbor, and the local updating rule $f$ is recorded as follows:

$$
f: S_{t}^{r} \rightarrow S_{t+1}
$$

The local updating rule is the input and output on a finite set of cells. The global evolution rule can be obtained by using the local updating rule for each cell in space:

$$
F\left(g_{t+1}^{i}\right)=f\left(g_{t}^{i-r}, \ldots, g_{t}^{i}, \ldots, g_{t}^{i+r}\right)
$$

where $g_{t}^{i}$ is the cell at position $i$ at the $t$-th time.

\subsection{Improved Cellular Bat Algorithm}

For the DERs aggregation model based on multi-scenario and multi-objective, this paper uses cellular bat algorithm based on fuzzy membership degree to solve it. Bat algorithm is a heuristic algorithm with good optimization characteristics. The principle of this algorithm is echolocation of the bat [24]. Compared with other heuristic algorithms, the cellular bat algorithm, which combines cellular automata with bat algorithm, has faster convergence speed and better global optimization ability. The optimal results obtained by this algorithm are the Pareto solution sets. In practical planning, only one planning scheme can be selected. Therefore, the optimal compromise solution can be selected according to the fuzzy membership degree [25].

Definition 1. Assume that the Euclidean space $H_{N^{*} D}$ is the search space of the bat population, the location of the $i$-th bat is as follows:

$$
U_{i}=\left\{u_{i 1}, \ldots u_{i j}, \ldots u_{i D} \mid u_{i j} \in\{0,1\}\right\}
$$

where $N$ is the total number of bats, $D$ is the code length or the dimension, $u_{i j}$ is the $j$-th code of the $i$-th bat location.

Definition 2. The distance between the $p$-th and the $q$-th bats is expressed as follows:

$$
\operatorname{dis}(p, q)=\sum_{j=1}^{D}\left|u_{p j}-u_{q j}\right| \quad p, q \in\{0,1, \ldots, N\}
$$

Definition 3. $U=\left(u_{1}, \ldots, u_{i}, \ldots, u_{D}\right)$ is a set, where $u_{i} \in\{0,1\}$, the arrangements of $u_{i}$ constitute the cell space, which is recorded as $L=\left\{\right.$ cellX $\left.=\left(u_{1}, \ldots u_{i}, \ldots u_{D}\right) \mid u_{i} \in\{0,1\}\right\}$, cellX is a cell composed of 0 and 1 arrays.

Definition 4. Multi-dimensional cell neighborhood type: $M=\{$ cellY $\mid$ dis (cellY-cellX $) \leq r$, cellX, cellY $\in$ L $\}$, where $r$ is the radius of the cellular neighbor, and $r=1$ is chosen in this paper.

Definition 5. Cell update rule: Determine the central cell and the neighbor cells according to the definition, calculate the target solution of the central cell and the neighbor cells, and select the cell corresponding to the optimal target solution. 
The steps of FMD-CBA are as follows:

(1) Initialize the parameters. Determine the bat population size $N$, the location $U_{i}^{0}$ of the $i$-th bat, the frequency $\beta_{i}^{0}$, the velocity $v_{i}^{0}$, and the pulse emission rate $R_{i}^{0}$. The initial codes of the $i$-th bat position are calculated as follows:

$$
u_{i j}^{0}=\left\{\begin{array}{l}
1, \operatorname{rand}() \geq 0.5 \\
0, \operatorname{rand}()<0.5
\end{array}\right.
$$

(2) Calculate the objective function value corresponding to each bat in the population.

(3) According to equations (25) and (26), update the speed of each bat in the population. The update formula of the bat location is as follows:

$$
\begin{gathered}
\beta_{i}=\beta_{\min }+\left(\beta_{\max }-\beta_{\min }\right) \gamma \\
v_{i}^{t+1}=v_{i}^{t}+\left(u_{i}^{t}-u_{o p}\right) \beta_{i} \\
u_{i j}^{t}=\left\{\begin{array}{l}
1,1 /\left(e^{-v_{i j}^{t}}+1\right) \geq \operatorname{rand}() \\
0,1 /\left(e^{-v_{i j}^{t}}+1\right)<\operatorname{rand}()
\end{array}\right.
\end{gathered}
$$

where $\beta_{i}, \beta_{\min }$ and $\beta_{\max }$ are the frequency, the minimum frequency and maximum frequency of the sound waves emitted of the $i$-th bat, $\gamma$ is a random number between 0 and $1, u_{o p}$ is the optimal location of the bat, $u_{i}^{t}$ and $v_{i}^{t}$ are the location and speed of the $i$-th bat in the $t$-th generation:

(4) If rand() $>R_{i}^{t}$, according to the definition of the multi-dimensional cell neighbor, update the cell in the neighbor range, and select the optimal solution $u^{t+1}$ in the neighbor. If not, regard the central cell as the optimal solution $u^{t+1}$, where $R_{i}^{t}$ is the emission rate of the $i$-th bat in the $t$-th generation.

(5) Calculate the objective function value $f^{t+1}$ corresponding to the optimal solution $u^{t+1}$. If $\operatorname{rand}()<A_{i}^{t}$ and $f^{t+1}$ dominate $f^{t}$, then accept the current solution, update $A^{t+1}$ and $R^{t+1}$ according to the formula.

(6) Determine whether the maximum number of iterations is reached, if it is, output the Pareto solution sets and go to the next step, otherwise return to step (3).

(7) Calculate the membership and weight of each objective function in the Pareto solution sets. Membership and weight are calculated as follows:

$$
\begin{gathered}
\varepsilon_{a b}=\frac{f_{b}^{\max }-f_{a b}}{f_{b}^{\max }-f_{b}^{\min }}, b=1,3,4 \\
\varepsilon_{a b}=\frac{f_{a b}-f_{b}^{\min }}{f_{b}^{\max }-f_{b}^{\min }}, b=2 \\
\omega_{b}=\frac{\sum_{c=1}^{N_{1}} \sum_{d=c}^{N_{1}}\left(\varepsilon_{c b}-\varepsilon_{d b}\right)^{2}}{\sum_{l=1}^{N_{2}} \sum_{c=1}^{N_{1}} \sum_{d=c}^{N_{1}}\left(\varepsilon_{c l}-\varepsilon_{d l}\right)^{2}}
\end{gathered}
$$

where $f_{a b}$ is the $b$-th objective function value for the group $a, f_{b}^{\max }$ and $f_{b}^{\min }$ are the maximum and minimum values of the $a$-th and $b$-th objective functions in the Pareto solution sets, $N_{1}$ is the number of optimal sets, $N_{2}$ is the number of objective functions. 
(8) Calculate the $S_{a}$ value corresponding to each solution, and take the solution corresponding to $S_{a \cdot \max }$ as the compromise optimal solution:

$$
S_{a}=\sum_{b=1}^{M} \varepsilon_{a b} \omega_{b}
$$

The flowchart of FMD-CBA is shown in Figure 1.

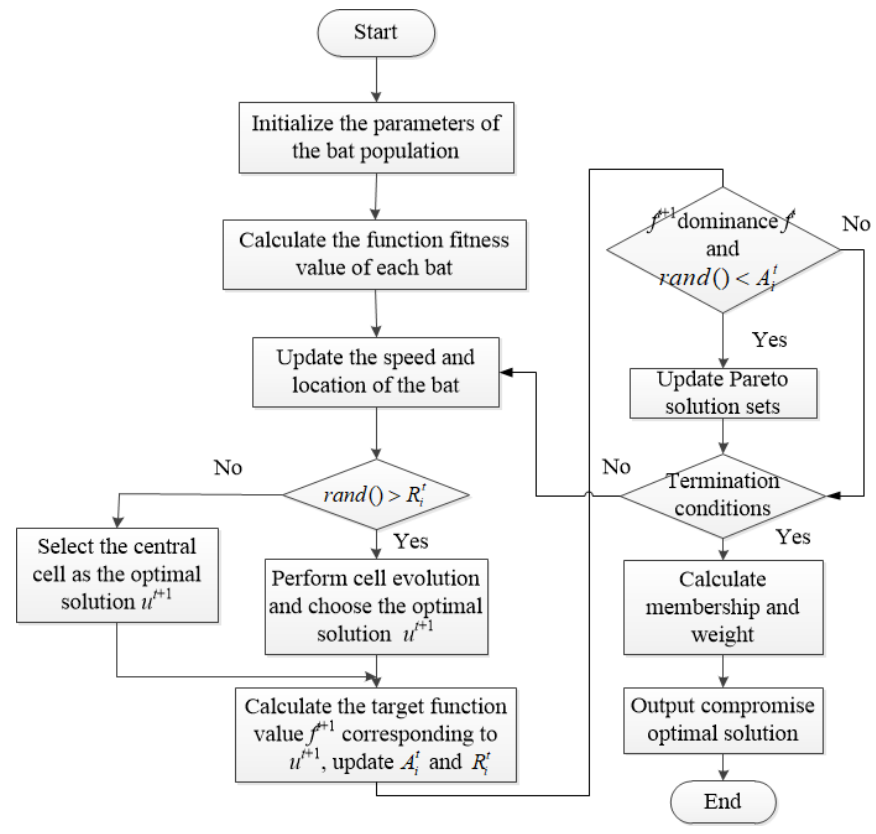

Figure 1. The flow chart of FMD-CBA.

\section{Example Analysis}

Taking an urban area in Hebei Province, China as an example, the season is set in the spring, the RA can be constructed through the reasonable selection of DERs. According to the distribution of the urban road network, the urban area is divided into 18 regions, as shown in Figure 2.

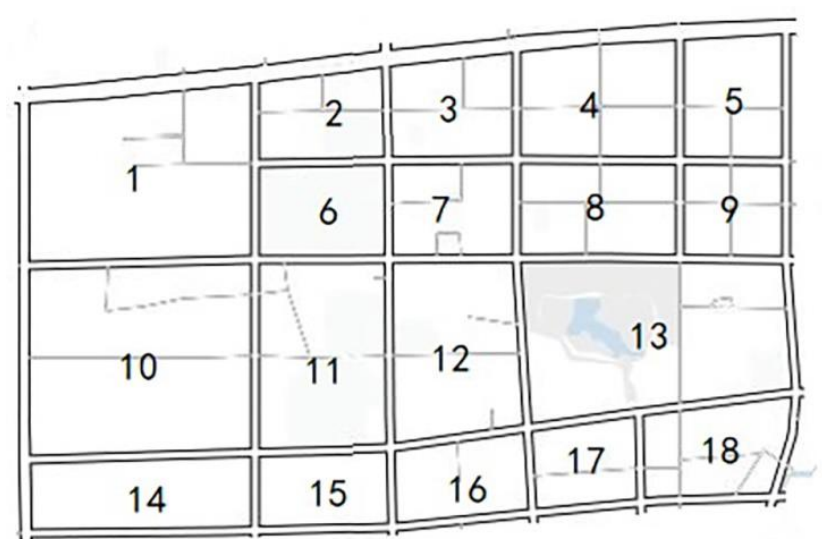

Figure 2. Location and number of each area.

The types of DERs in each region are different. $X_{n}^{D G}, X_{n}^{E S}$ and $X_{n}^{D R}$ represent the existence of DG, ES and DR in the $n$-th region respectively, 1 is the existence and 0 is the non-existence. The types of DERs in 18 regions are shown in Table 1. 
Table 1. Distribution of resources in each region.

\begin{tabular}{ccccccccccccccccccc}
\hline Number & $\mathbf{1}$ & $\mathbf{2}$ & $\mathbf{3}$ & $\mathbf{4}$ & $\mathbf{5}$ & $\mathbf{6}$ & $\mathbf{7}$ & $\mathbf{8}$ & $\mathbf{9}$ & $\mathbf{1 0}$ & $\mathbf{1 1}$ & $\mathbf{1 2}$ & $\mathbf{1 3}$ & $\mathbf{1 4}$ & $\mathbf{1 5}$ & $\mathbf{1 6}$ & $\mathbf{1 7}$ & $\mathbf{1 8}$ \\
\hline DG & 1 & 1 & 1 & 1 & 1 & 1 & 1 & 1 & 1 & 1 & 1 & 1 & 1 & 1 & 0 & 1 & 1 & 1 \\
ES & 0 & 1 & 1 & 1 & 0 & 1 & 1 & 0 & 0 & 1 & 1 & 1 & 0 & 1 & 0 & 0 & 1 & 1 \\
DR & 0 & 1 & 1 & 1 & 1 & 1 & 1 & 0 & 0 & 1 & 0 & 1 & 0 & 1 & 0 & 1 & 1 & 1 \\
\hline
\end{tabular}

In Table 1 , the DG in regions 1 and 13 are WP, and the DG in other regions is PV. In DG, the generation characteristics of WP and PV are mainly considered. In ES, the power characteristics of electric vehicle are mainly considered. In DR, response characteristics of demand resource are mainly considered, which can also be regarded as virtual output characteristics.

As can be seen from Table 1, there are 17 regions with DG, of which 15 regions are WP and 2 regions are PV. In the process of multi-scenario construction, according to the 92-day WP and PV output curves in the 17 regions in spring, the feature indexes are extracted, and 92 clustering vectors containing the output characteristics of each region are obtained, of which the dimensions of the vector are 68. The improved K-means algorithm is used to cluster 92 eigenvectors in spring. The relationship between the number of clusters and the contour coefficients is shown in Figure 3.

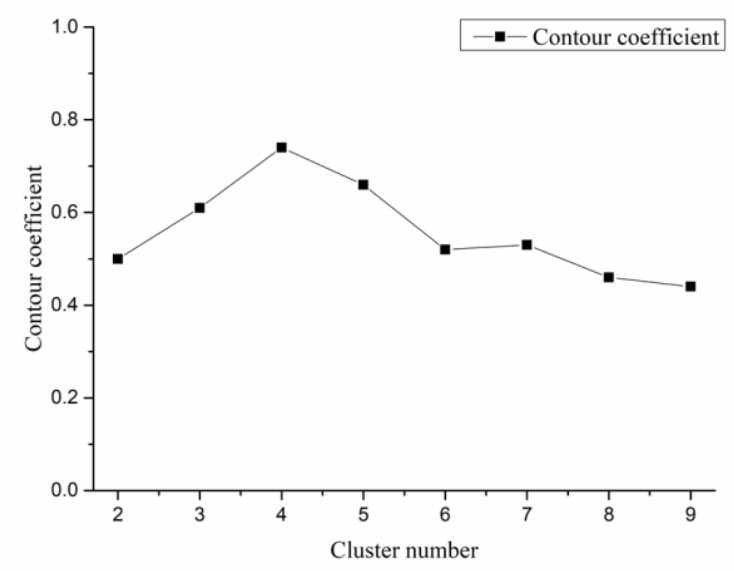

Figure 3. The curve of cluster number and contour coefficient.

The number of clusters is set to 2-9. By calculating the contour coefficients corresponding to the number of different clusters, the optimal cluster number is 4 and the maximum contour coefficient is 0.74. The number 1-92 are used to represent the 1-92th days of spring. The ratio of the number of days in each scenario and the total number of days represents the probability of the scenario. The probability and the corresponding days are shown in Table 2.

Table 2. The results of multi-scenario division.

\begin{tabular}{ccc}
\hline Scenario & Scenario Probability & Date Number \\
\hline 1 & $21 / 92$ & $1-16,18,20,22,27,29$ \\
2 & $23 / 92$ & $17,19,21,23-25,26,28,30-39,41,42,44,45,49$, \\
3 & $26 / 92$ & $40,43,46-48,50-63,65,66,68,71,73-75$ \\
4 & $24 / 92$ & $64,67,69,70,72,76-92$ \\
\hline
\end{tabular}


Taking the WP in the 1st region and the PV in the 4th region as an example, the typical output curve and daily output curves in each scenario are plotted. The typical output value at each time is expressed by the average output value of all days, as shown in Figures 4 and 5.

By comparing the typical output curves in the four scenarios, it can be seen that the curves are significantly different. The typical output of WP decreases as a whole, and the typical output of PV increases as a whole. This trend is in line with the trend of wind speed decreasing and solar irradiance increasing gradually from the beginning of spring to the end of spring. The parameters of FMD-CBA are set as follows: the size of the bat population is 60 , the number of iterations is 100 , and the initial emission rate is 0 . In order to verify the effectiveness of the algorithm, the bat algorithm (BA) and ant colony algorithm (ACA) are added to compare the optimal results with CBA, and the numbers of populations and iterations are same. The convergence curves of the three algorithms are shown in Figure 6. In the early stage of iteration, the convergence characteristics of the three algorithms are basically the same. However, with the increase of iteration times, the number of Pareto solution sets searched by CBA is obviously more than that of the other two algorithms. CBA, BA and ACA reached convergence at the 48th, 58th and 62nd iteration times respectively, and the final number of Pareto solution sets is 121. It shows that the CBA has the advantage of speed.

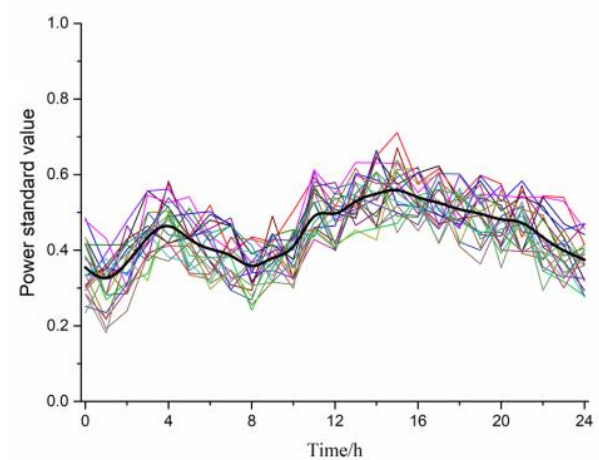

(a)

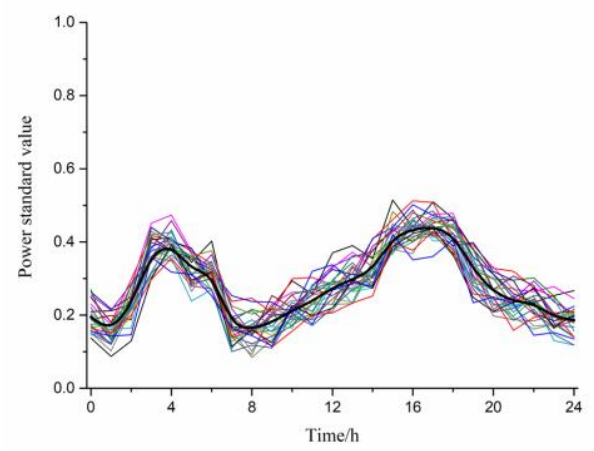

(c)

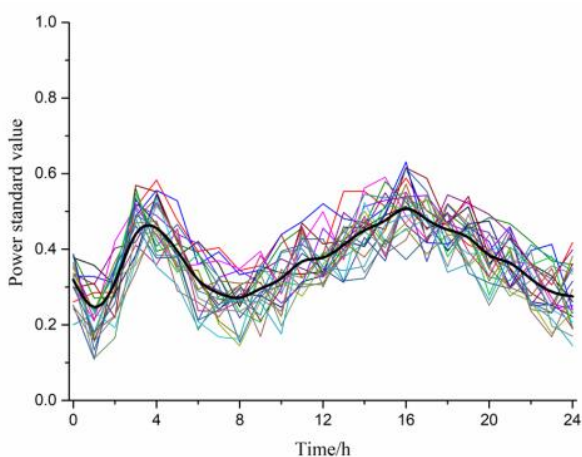

(b)

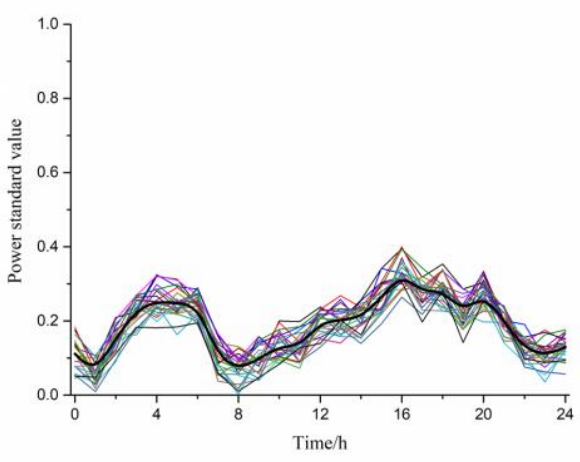

(d)

Figure 4. Wind power generation in typical scenarios (a) Wind power generation in scenario 1 ; (b) Wind power generation in scenario 2; (c) Wind power generation in scenario 3; (d) Wind power generation in scenario 4. 


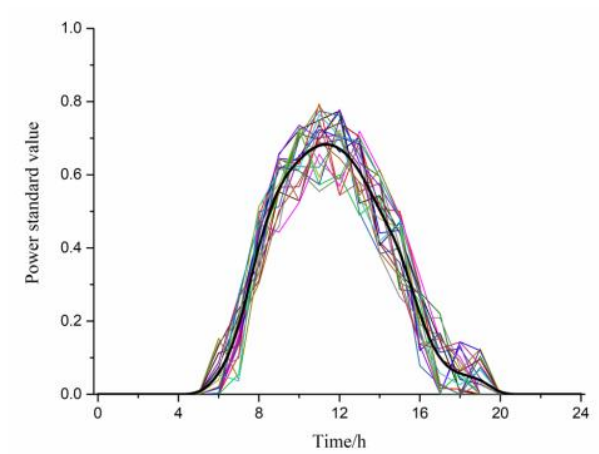

(a)

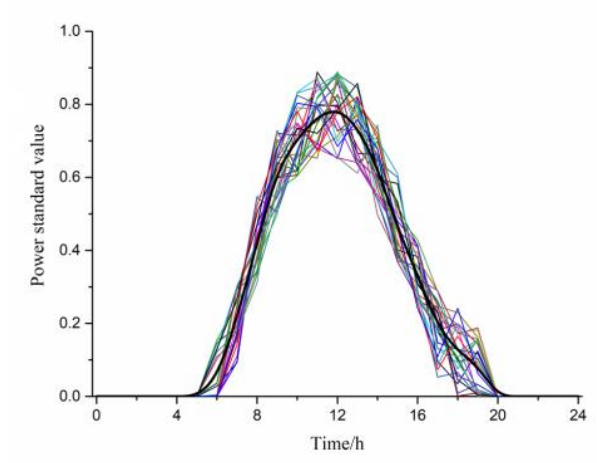

(c)

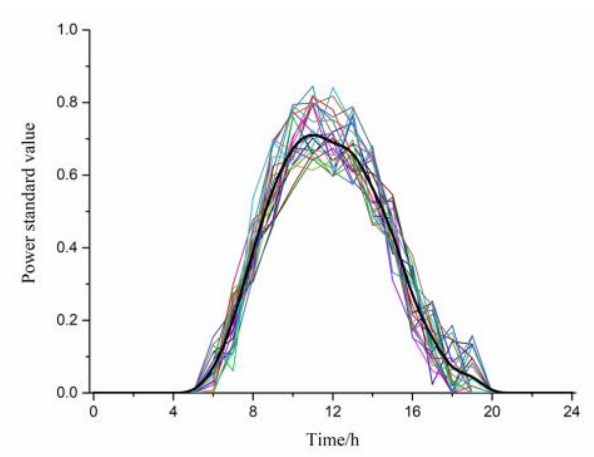

(b)

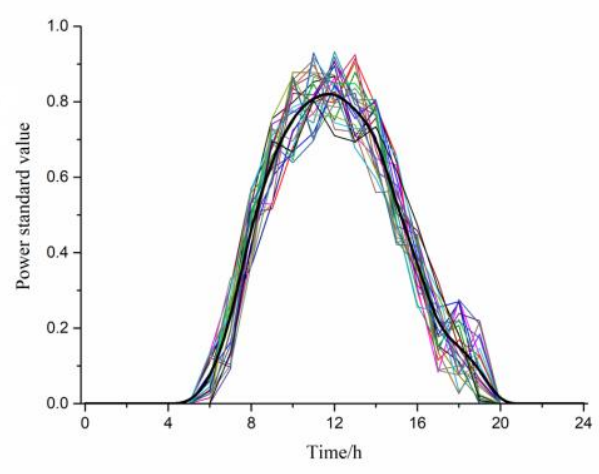

(d)

Figure 5. Photovoltaic generation in typical scenarios (a) Photovoltaic generation in scenario 1; (b) Photovoltaic generation in scenario 2; (c) Photovoltaic generation in scenario 3; (d) Photovoltaic generation in scenario 4 .

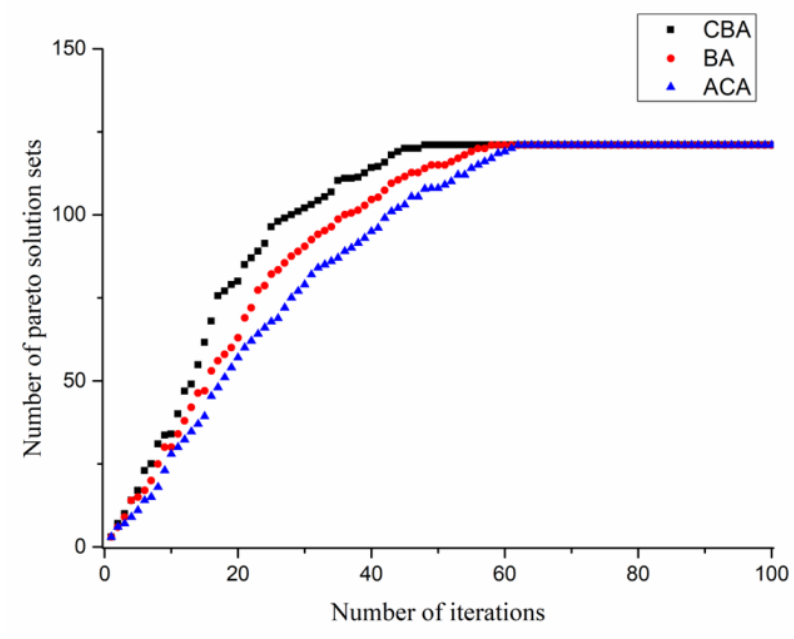

Figure 6. Iterative convergence process.

The typical output and the probability of each scenario in the spring of each region are substituted into the multi-objective DERs aggregation model, and FMD-CBA is used to select the resources of each region. The obtained Pareto solution sets are shown in Figure 7. 


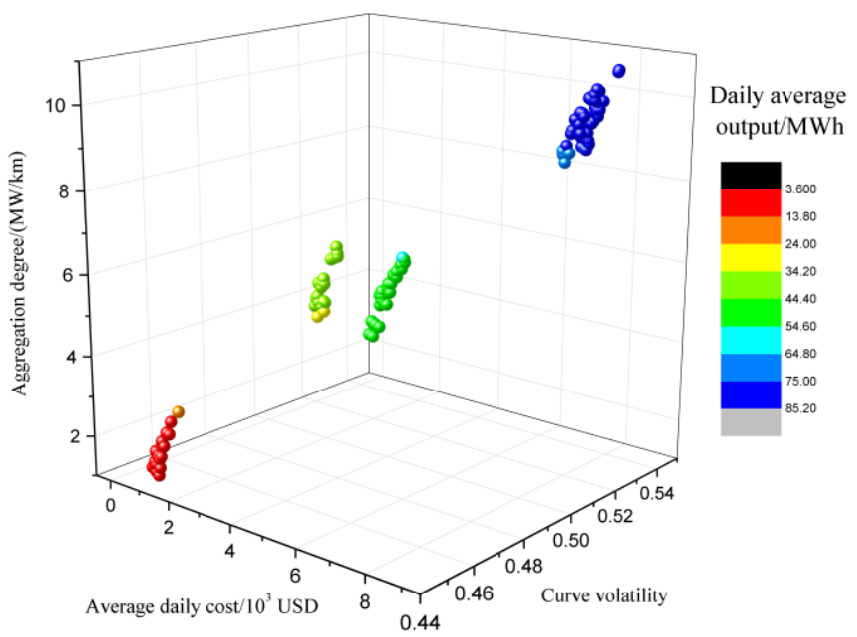

Figure 7. Pareto solution sets.

The Pareto solution sets obtained by FMD-CBA take into account the global search and the local search. The number of the Pareto solution sets is 121 . But in practice, only one planning scheme is adopted when constructing a RA. Therefore, this paper calculates the membership and weight of each objective function through fuzzy compromise programming and then obtains a compromise solution. The compromise solution and the value range of each objective function are shown in Table 3.

Table 3. The value of each objective function.

\begin{tabular}{ccccc}
\hline & $f_{\mathbf{1}} \mathbf{( \mathbf { 1 0 } ^ { \mathbf { 3 } } \mathbf { U S D } )}$ & $f_{\mathbf{2}} \mathbf{( M W h )}$ & $f_{\mathbf{3}}$ & $f_{\mathbf{4}} \mathbf{( \mathbf { M W } / \mathbf { k m } )}$ \\
\hline Maximum value & 9.14 & 79.14 & 0.54 & 10.58 \\
Minimum value & 0.48 & 3.8 & 0.45 & 1.04 \\
Compromise Solution & 5.18 & 46.5 & 0.49 & 6.22 \\
\hline
\end{tabular}

Under this compromise solution, the average daily cost is 5180 USD, the average daily output is $46.5 \mathrm{MWh}$, the volatility is 0.49 , and the aggregation degree is $6.22 \mathrm{MW} / \mathrm{km}$. The corresponding aggregation scheme of the compromise solution is shown in Table 4. ' $\checkmark$ ' represents that the resource participates in the aggregation, ' $x$ ' represents that the resource does not participate in the aggregation, and the blank represents that there are no such resources in this region.

Table 4. Final aggregation scheme.

\begin{tabular}{ccccccccccccccccccc}
\hline Number & 1 & 2 & 3 & 4 & 5 & 6 & 7 & 8 & 9 & 10 & 11 & 12 & 13 & 14 & 15 & 16 & 17 & 18 \\
\hline DG & $\times$ & $\times$ & $\sqrt{ }$ & $\sqrt{ }$ & $\times$ & $\sqrt{ }$ & $\sqrt{ }$ & $\sqrt{ }$ & $\sqrt{ }$ & $\sqrt{ }$ & $\sqrt{ }$ & $\sqrt{ }$ & $\sqrt{ }$ & $\sqrt{ }$ & & $\sqrt{ }$ & $\sqrt{ }$ & $\times$ \\
ES & & $\times$ & $\sqrt{ }$ & $\sqrt{ }$ & & $\sqrt{ }$ & $\sqrt{ }$ & & & $\times$ & $\sqrt{ }$ & $\times$ & & $\times$ & & & $\times$ & $\sqrt{ }$ \\
DR & & $\sqrt{ }$ & $\sqrt{ }$ & $\times$ & $\sqrt{ }$ & $\sqrt{ }$ & $\sqrt{ }$ & & & $\sqrt{ }$ & & $\sqrt{ }$ & & $\sqrt{ }$ & & $\sqrt{ }$ & $\sqrt{ }$ & $\sqrt{ }$ \\
\hline
\end{tabular}

The final aggregation scheme includes DG in 13 regions (WP in 1 region and PV in 12 regions), ES in 6 regions and DR in 11 regions. The aggregation scheme corresponds to the compromise solution which considers cost, output characteristics and aggregation degree. The actual output curve of the RA is superimposed by the curves of WP, PV, ES and DR, as shown in Figure 8. 


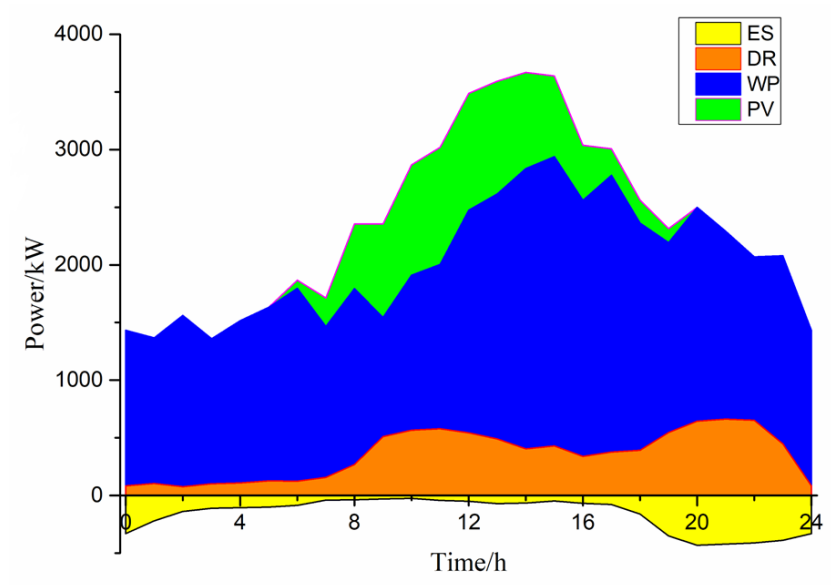

Figure 8. Output Curve of RA.

The area of various DERs represents the daily average output value. WP is $35.06 \mathrm{MWh}, \mathrm{PV}$ is 7.14 MWh, DR is 7.6 MWh, ES is $-3.3 \mathrm{MWh}$, and the daily average output of the RA is $46.5 \mathrm{MWh}$. From Figure 8, DG accounts for the majority of the RA output. Although the capacity of ES and DR is small, the output curve of the RA can be adjusted.

To verify the validity of the single-season aggregation model proposed in this paper, the aggregation results of multi-scenario in other seasons are given in Table 5. And DER $\mathrm{n}$ represents the resource type in the $n$-th region. The number of aggregated resources of multi-scenario in summer is 33 . Due to the increase of solar irradiance and the decrease of wind speed from spring to summer, the aggregation results increase photovoltaic resources in two regions and energy storage resources in one region. Due to the decrease of solar irradiance and the increase of wind speed from summer to autumn, the aggregation results decrease wind power resources in two regions, increase energy storage resources in one region and decrease demand resources in one region. Due to the decrease of solar irradiance and the increase of wind speed from autumn to winter, the aggregation results increase wind power resources in one region, decrease energy storage resources in two regions and increase demand resources in one region. Compared with the multi-scenario in spring, with the increase of resource utilization, the average daily cost, average daily output, volatility and aggregation degree all increase. Affected by the changes in solar irradiance and wind speed, the aggregation results in autumn and winter are also different from those in spring. That is, the aggregation results in different seasons are different, which verifies the validity of the single-season aggregation model.

To verify the validity of the multi-scenario aggregation model proposed in this paper, the single-scenario aggregation results in spring and in a year are given in Table 6 . In the single-scenario aggregation model in spring, this paper only considers the existence of scenario 1 , that is, $p_{1}=1$, $p_{2}=p_{3}=p_{4}=0$. Compared with the multi-scenario in spring, the number of aggregated resources in single scenario 1 is 29 . Although the volatility is slightly smaller, it is inferior to multi-scenario in terms of daily average cost, daily average output and aggregation degree. In the single-scenario aggregation model in a year, the annual average output curves are used to replace the output of DG. Compared with multi-scenario in spring, although the daily average output is larger, it is inferior to multi-scenarios in other aspects. Therefore, it is better to consider the aggregation results of multiple-scenarios. 
Table 5. Resource aggregation results in each season.

\begin{tabular}{ccccc}
\hline & $\begin{array}{c}\text { Multi-Scenario in } \\
\text { Spring }\end{array}$ & $\begin{array}{c}\text { Multi-Scenario in } \\
\text { Summer }\end{array}$ & $\begin{array}{c}\text { Multi-Scenario in } \\
\text { Autumn }\end{array}$ & $\begin{array}{c}\text { Multi-Scenario in } \\
\text { Winter }\end{array}$ \\
\hline $\begin{array}{c}\mathrm{DG}_{1}, \mathrm{DG}_{2}, \mathrm{DG}_{5}, \mathrm{DG}_{18}, \\
\mathrm{ES}_{2}, \mathrm{ES}_{10}, \mathrm{ES}_{12}, \mathrm{ES}_{14}, \\
\mathrm{ES}_{17}, \mathrm{DR}_{4}\end{array}$ & $\begin{array}{c}\mathrm{DG}_{1}, \mathrm{DG}_{18}, \mathrm{ES}_{2}, \mathrm{ES}_{7}, \\
\mathrm{ES}_{12}, \mathrm{ES}_{17}, \mathrm{DR}_{4}\end{array}$ & $\begin{array}{r}\mathrm{DG}_{1}, \mathrm{DG}_{2}, \mathrm{DG}_{10}, \\
\mathrm{DG}_{18}, \mathrm{ES}_{2}, \mathrm{ES}_{10}, \mathrm{ES}_{17}, \\
\mathrm{DR}_{4}, \mathrm{DR}_{18}\end{array}$ & $\begin{array}{c}\mathrm{DG}_{2}, \mathrm{DG}_{5}, \mathrm{DG}_{14}, \\
\mathrm{DG}_{18}, \mathrm{ES}_{2}, \mathrm{ES}_{4}, \mathrm{ES}_{14}, \\
\mathrm{ES}_{17}, \mathrm{ES}_{18}, \mathrm{DR}_{5}\end{array}$ \\
$\begin{array}{c}\text { Number of } \\
\text { aggregated resources } \\
\text { Average daily cost } \\
\quad\left(10^{3} \text { USD) }\right.\end{array}$ & 30 & 33 & 31 & 30 \\
$\begin{array}{c}\text { Average daily } \\
\text { output (MWh) }\end{array}$ & 5.16 & 6.33 & 6.05 & 5.74 \\
$\begin{array}{c}\text { Curve volatility } \\
\text { Aggregation degree } \\
\text { (MW/km) }\end{array}$ & 46.5 & 59.16 & 55.42 & 50.25 \\
Resource utilization rate & 0.49 & 0.53 & 0.51 & 0.5 \\
\hline
\end{tabular}

Table 6. Single-scenario aggregation results.

\begin{tabular}{cccc}
\hline & Multi-Scenario in Spring & Single-Scenario in Spring & Single-Scenario in a Year \\
\hline \multirow{2}{*}{ Unaggregated resources } & $\mathrm{DG}_{1}, \mathrm{DG}_{2}, \mathrm{DG}_{5}, \mathrm{DG}_{18}, \mathrm{ES}_{2}$, & $\mathrm{DG}_{1}, \mathrm{DG}_{5}, \mathrm{DG}_{9}, \mathrm{DG}_{18}, \mathrm{ES}_{2}$, & $\mathrm{DG}_{5}, \mathrm{DG}_{10}, \mathrm{DG}_{18}, \mathrm{ES}_{4}, \mathrm{ES}_{10}$, \\
& $\mathrm{ES}_{10}, \mathrm{ES}_{12}, \mathrm{ES}_{14}, \mathrm{ES}_{17}, \mathrm{DR}_{4}, \mathrm{ES}_{17}, \mathrm{DR}_{4}$, & $\mathrm{ES}_{14}, \mathrm{ES}_{18}, \mathrm{DR}_{5}, \mathrm{DR}_{10}$ \\
Number of aggregated resources & 30 & $\mathrm{DR}_{5}$ & 31 \\
Average daily cost $\left(10^{3} \mathrm{USD}\right)$ & 5.18 & 29 & 6.28 \\
Average daily output $(\mathrm{MWh})$ & 46.5 & 5.41 & 50.23 \\
Curve volatility & 0.49 & 43.01 & 0.54 \\
Aggregation degree $(\mathrm{MW} / \mathrm{km})$ & 6.22 & 0.46 & 7.52 \\
Resource utilization rate & 0.78 & 7.69 & 0.76 \\
\hline
\end{tabular}

\section{Conclusions}

Aiming at the problem that the aggregation scheme obtained in a longer time scale can not achieve good resource aggregation effect, this paper establishes a single-season DERs aggregation model. Firstly, the daily eigenvectors of DG are extracted, and the contour coefficients are introduced. According to the improved K-means algorithm, the multiple probability scenarios in a single-season are obtained. Then, considering the daily average cost, daily output, curve volatility and aggregation degree, a DERs aggregation model based on multi-scenario and multi-objective is established, and FMD-CBA is used to solve the model.

According to the example analysis, the following conclusions can be obtained: (1) It is feasible to obtain scenarios with different probability by the clustering algorithm, and the typical output curves of WP and PV in each scenario have obvious differences. (2) The validity of the aggregation model based on single-season and multi-scenario is reflected in two aspects: one is that the aggregation results of different seasons are different, and the other is that the aggregation results of multi-scenario partition are better than that of single-scenario partition. (3) By choosing the Pareto solution sets through fuzzy compromise programming, an optimal compromise scheme can be obtained, which can provide a reference for planners.

In future work, the risk loss caused by the uncertainty of DERs can be considered and it also can be planned as a constraint. Traditional power plants can be added to the aggregation model and the environmental impact of thermal power can be considered. In addition, the study of single RA can be extended to multiple RAs. Through the establishment of the corresponding index evaluation system, it will be determined that several RAs should be established in the whole region.

Author Contributions: The author J.D. carried out the main research tasks and wrote the full manuscript, and H.L. proposed the original idea, analyzed the results and the whole manuscript. D.Z. and H.L. contributed to data processing and writing, while J.Y. made a language revision.

Funding: The authors would like to acknowledge funding by the National Natural Science Foundation of China (51607068).

Conflicts of Interest: The authors declare no conflict of interest. 


\section{Abbreviations}

The following abbreviations are used in this manuscript.

$\begin{array}{ll}\text { DERs } & \text { Distributed energy resources } \\ \text { RA } & \text { Resource aggregation } \\ \text { DG } & \text { Distributed generation } \\ \text { DR } & \text { Demand resource } \\ \text { ES } & \text { Energy storage } \\ \text { FMD-CBA } & \text { Cellular bat algorithm based on fuzzy membership degree } \\ \text { WP } & \text { Wind power } \\ \text { PV } & \text { Photovoltaic }\end{array}$

\section{References}

1. Babaei, S.; Zhao, C.; Fan, L.; Liu, T. Incentive-Based Coordination Mechanism for Renewable and Conventional Energy Suppliers. IEEE Trans. Power Syst. 2018, 34, 1761-1770. [CrossRef]

2. Gouveia, J.P.; Dias, L.; Martins, I.; Seixas, J. Effects of renewables penetration on the security of Portuguese electricity supply. Appl. Energy 2014, 123, 438-447. [CrossRef]

3. Abadi, S.; Davarpanah, M.; Mahmoodi, M.; Nasiri, S.; Bekhradian, R. Multi-objective Optimal DERs Placement and Sizing Considering Generator Shaft Fatigue. IEEE Trans. Power Syst. 2018, 33, 6787-6794. [CrossRef]

4. Trovato, V.; Sanz, I.; Chaudhuri, B.; Strbac, G. Preventing cascading tripping of distributed generators during non-islanding conditions using thermostatic loads. Int. J. Electr. Power Energy Syst. 2019, 106, $183-191$. [CrossRef]

5. Seritan, G.; Porumb, R.; Cepisca, C.; Grigorescu, S.D. Electricity distribution-Intelligent Solutions for Electricity Transmission and Distribution Networks. In Integration of Dispersed Power Generation; Series: Energy Systems; Karampelas, P., Ekonomu, L., Eds.; Springer: Berlin/Heidelberg, Germany, 2016; pp. $27-61$.

6. Ali, E.; Qiang, Y. Optimal integration and planning of renewable distributed generation in the power distribution networks: A review of analytical techniques. Appl. Energy 2018, 210, 44-59.

7. Asmus, P. Microgrids, virtual power plants and our distributed energy future. Electr. J. 2010, 23, 72-82. [CrossRef]

8. $\quad$ Eid, C.; Codani, P.; Perez, Y.; Reneses, J.; Hakvoort, R. Managing electric flexibility from Distributed Energy Resources: A review of incentives for market design. Renew. Sustain. Energy Rev. 2016, 64, 237-247. [CrossRef]

9. Calvillo, C.F.; Sánchez-Miralles, A.; Villar, J.; Martín, F. Optimal planning and operation of aggregated distributed energy resources with market participation. Appl. Energy 2016, 182, 340-357. [CrossRef]

10. Juan, M.; Mahdi, P.; Matti, L.; José, R. Optimal location-allocation of storage devices and renewable-based dg in distribution systems. Electr. Power Syst. Res. 2019, 172, 11-21.

11. Wu, L.; Jiang, L.; Hao, X. Optimal Scenario Generation Algorithm for Multi-objective Optimization Operation of Active Distribution Network. In Proceedings of the 2017 36th Chinese Control Conference, Dalian, China, 26-28 July 2017; pp. 2680-2685.

12. Zou, Y.; Yang, L. Synergetic dispatch models of a wind/PV/hydro virtual power plant based on representative scenario set. Power Syst. Technol. 2015, 39, 1855-1859.

13. Liu, Y.; Yang, J.; Tang, Y.; Xu, J.; Sun, Y.; Chen, Y.; Peng, X.; Liao, S. Bi-level fuzzy stochastic expectation modelling and optimization for energy storage systems planning in virtual power plants. J. Renew. Sustain. Energy. 2019, 11, 014101. [CrossRef]

14. Marco, P.; Stefano, R.; Daniele, M. A virtual power plant architecture for the demand-side management of smart prosumers. Appl. Sci. 2018, 8, 432.

15. Lujie, H.; Ping, L.; Sheng, C.; Qiaoyong, C. The Load Aggregation Strategy of Central Air-conditioning for Smoothing Wind Power Fluctuation. In Proceedings of the 2018 37th Chinese Control Conference, Wuhan, China, 28-27 July 2018; pp. 8870-8875.

16. Wang, Y.; Bian, H.; Wang, C. Research on Charging and Discharging Dispatching Strategy for Electric Vehicles. Open Fuels Energy Sci. J. 2015, 8, 176-182. 
17. Hrvoje, P.; Igor, K.; Tomislav, C. Virtual power plant mid-term dispatch optimization. Appl. Energy 2013, 101, 134-141.

18. Xiong, H.; Wu, J.; Chen, J. K-Means Clustering Versus Validation Measures: A Data-Distribution Perspective. IEEE Trans. Syst. Man Cybern. Part B 2008, 39, 318-331. [CrossRef] [PubMed]

19. Zhang, Y.; Liu, N.; Wang, S.; Asghar, M.Z. A differential privacy protecting K-means clustering algorithm based on contour coefficients. PLoS ONE 2018, 13, e0206832. [CrossRef] [PubMed]

20. Yan, T.; QU, Z.; Hui, D. Economic analysis of the virtual power plants with large-scale battery energy storage systems. Autom. Electr. Power Syst. 2014, 38, 98-104.

21. Porumb, R.; Postolache, P.; Seritan, G.; Vatu, R.; Ceaki, O. Load profiles analysis for electricity market. Comput. Methods Soc. Sci. 2013, 1, 30-38.

22. Wei, G.; Junyong, L.; Xingqi, H.E.; Yang, L. Load Restoration Considering Load Fluctuation Rate and Load Complementary Coefficient. Power Syst. Technol. 2014, 38, 2490-2496.

23. Sirakoulis, G.C.; Karafyllidis, I.; Thanailakis, A. A CAD system for the construction and VLSI implementation of Cellular Automata algorithms using VHDL. Microprocess. Microsyst. 2003, 27, 381-396. [CrossRef]

24. Ghanem, W.; Jantan, A. An enhanced Bat algorithm with mutation operator for numerical optimization problems. Neural Comput. Appl. 2019, 31, 617-651. [CrossRef]

25. Chowdhary, C.L.; Acharjya, D.P. Clustering Algorithm in Possibilistic Exponential Fuzzy C-Mean Segmenting Medical Images. J. Biomim. Biomater. Biomed. Eng. 2017, 30, 12-23. [CrossRef]

(C) 2019 by the authors. Licensee MDPI, Basel, Switzerland. This article is an open access article distributed under the terms and conditions of the Creative Commons Attribution (CC BY) license (http://creativecommons.org/licenses/by/4.0/). 